\title{
NanoMi: An Open Source (Scanning) Transmission Electron Microscope.
}

Marek Malac ${ }^{1,2}$, Martin Cloutier ${ }^{1}$, Mark Salomons ${ }^{1}$, Sean Chen ${ }^{3}$, Suliat Yakubu ${ }^{3}$, Marcus Leeson ${ }^{3}$, Jason Pitters $^{1}$, Doug Vick ${ }^{1}$, Drew Price ${ }^{1}$, Darren Homeniuk ${ }^{1}$, Misa Hayashida ${ }^{4}$ and Ray Egerton ${ }^{2}$

${ }^{1}$ NRC-NANO, Edmonton, Alberta, Canada, ${ }^{2}$ Department of Physics, University of Alberta, Edmonton, Alberta, Canada, ${ }^{3}$ University of British Columbia, Vancouver, British Columbia, Canada, ${ }^{4}$ National Research Council Canada, Edmonton, Alberta, Canada,

We are developing a modular (scanning) transmission electron microscope (S)TEM, referred to as NanoMi, which is released under an open source license [1] by the National Research Council, Canada; see [2] for updates.

The electron microscope (EM) is a critical enabling tool in the physical, materials and biological sciences but its impact could be increased by the availability of an easy-to-build, customizable instrument capable of modest resolution and suitable for electron beam lithography, specialized experiments and for training personnel. An instrument that can be built for $\sim 50 \mathrm{k} \$$ and $\sim 1$ year of student time would fulfill these requirements in many laboratories around the world. Here we describe an initial design of such an instrument.

NanoMi consists of an electrostatic Einzel lens (EL) [3] column suspended in a vacuum chamber (Fig. 1), based on widely available ConFlat ${ }^{\mathrm{TM}}(\mathrm{CF})$ vacuum hardware $(\mathrm{HW})$ and off-the-shelf high-voltage power supplies. The use of standard CF HW decreases the cost and enables ultra high vacuum (UHV) operation for surface-science experiments. The separation of the electron optics from the vacuum envelope, together with column modular design, allows NanoMi to be used to study EM principles and as a test bench for electro-optical elements, including electron sources, spectrometers, aberration correctors, ultrafast pumpand-probe experiments etc. The electrostatic column can be adapted for use with ion beams.

The probe-forming section of the column can be used independently as an UHV-compatible SEM or an electron-beam lithography tool. Although it may limit imaging performance, the use of EL (Fig 2) simplifies manufacturing and eliminates the need for water cooling, NanoMi is currently equipped with a JEOL-1400 gun with W-hairpin or LaB 6 filament and the probe-forming system contains three condenser lenses, allowing $\sim 1300 \mathrm{x}$ demagnification of the electron source. The imaging system is designed with five lenses in addition to an objective, resulting in over 50,000x maximum screen magnification, Fig 3 . The modular design allows any lens to be replaced by a permanent magnet lens, to improve the spatial resolution.

NanoMi target specifications are: STEM and SEM probe size $\sim 10 \mathrm{~nm}$, TEM image resolution $\sim 5 \mathrm{~nm}$ and diffraction with $10-100 \mathrm{~cm}$ camera length at $50 \mathrm{kV}$ incident electron energy. The use of a low acceleration voltage limits the thickness of samples that can be examined in (S)TEM mode but can be advantageous for study of radiation-sensitive materials. In TEM mode, we utilize a Sony $\alpha 6000$ camera with a fixed 50mm lens imaging a scintillator screen, Fig 4. NanoMi is controlled from a single computer that provides DAC control. An in-house piezo mechanism is used for sample stage and aperture movement, allowing sub-nm lateral positioning of $3 \mathrm{~mm}$ diameter discs for the apertures and sample. The column has a port for an ion pump, although a single turbomolecular pump (TMP) is sufficient for Whairpin or $\mathrm{LaB} 6$ operation and provides $\sim 5 \times 10^{-8}$ torr vacuum at the sample plane [4]. 


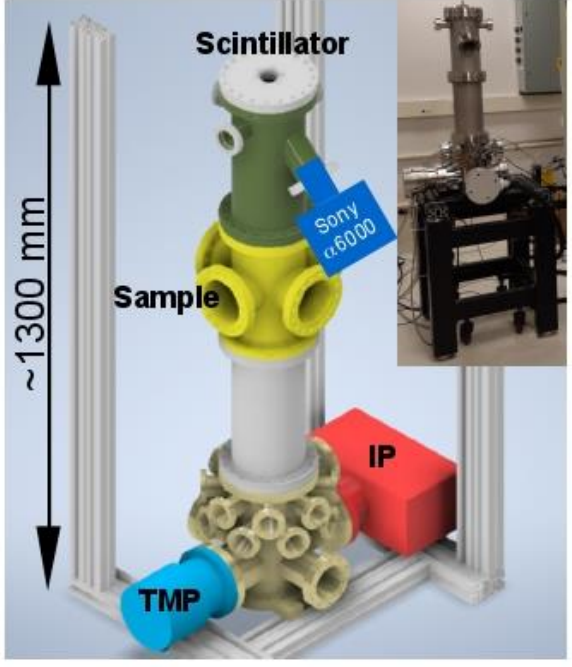

Fig 1. NanoMi column is based on 8" CF hardware The electron source is placed near a turbopump (TMP) and electrical feedthroughs. A 6-way CF cross houses the sample transfer. Detectors are located at the top of the column. An ion pump can be installed when field emission gun is utilized. Inset: photo of the instrument.

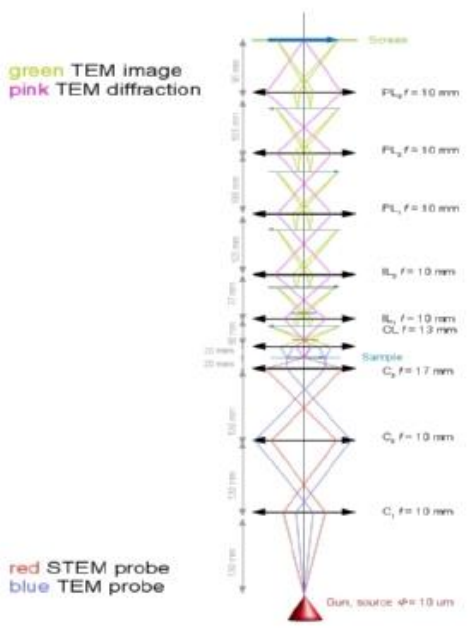

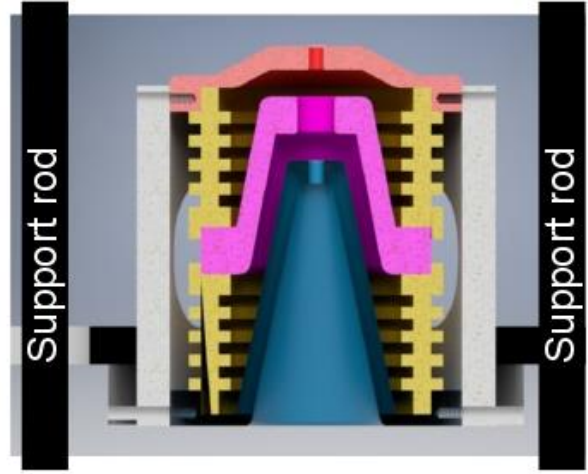

Fig 2. NanoMi Einzel lens. Biased electrode (pink) is placed between two grounded electrodes (blue and red) separated by an insulator (yellow). The entire assembly is placed inside a stainless steel puck with mounts (gray). The column can be composed either of identical lens or various optical el ements mounted on support rods (black). The stainless steel puck is about $50 \mathrm{~mm}$ tall and about $50 \mathrm{~mm}$ diameter.

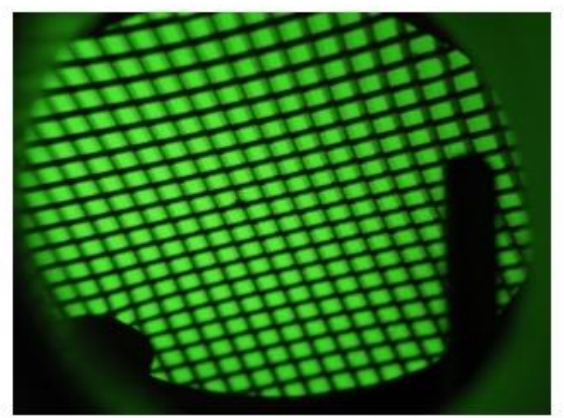

Fig 4. The first image obtained in NanoMi. A 200 mesh $\mathrm{Cu}$ grid placed between an electron source and a single Einzel lens was magnified about $100 \mathrm{x}$ suggesting focal length $\sim 10 \mathrm{~mm}$ at incident energy $\sim 15 \mathrm{kV}$

Fig 3. Approximate layout of NanoMi optics It consists of three probe-forming lens, objective, two intermediate lens and two or three projector lens.

\section{Figure 1. NanoMi}

\section{References}

[1] https://www.gnu.org/licenses/old-licenses/gpl-2.0.en.html

[2] https://nrc.canada.ca/en/, www.tem-eels.ca and www.msc-smc.org

[3] G. Rempfer, J. Appl. Phys. 57 (1985), p. 2385.

[4] The support and encouragement of Brian Legge (JEOL Canada), Dr. Y. Taniguchi (Hitachi High Tech. Corp.), Dr. S. Motoki (JEOL Ltd), D. Hoyle (Hitachi High Tech.Canada) Dr. Y. Nagatani (JPARC, Japan), Dr. H. Okamoto (Akita Pref. U., Japan), Dr. M. Marko (Wadsworth Centre, New York, USA), Prof. M. Freeman (U of Alberta), Prof. M. Beleggia (Denmark, Tech. U) as well as many others made this project possible. 